\title{
The neuropsychiatry
}

\section{of brain tumours}

Piet Oosthuizen, MB ChB, MMed (Psych), PhD Department of Psychiatry, Stellenbosch University, Tygerberg, W Cape

Every psychiatrist who has worked in the clinical field for some time will be able to relate a story of a patient who presented with psychiatric symptoms but eventually turned out to have a brain tumour. We all fear that someday we will misdiagnose a brain tumour and therefore fail to save a patient's life. The purpose of this article is to give a brief outline of the important clinical issues related to brain tumours and psychiatry.

The mere fact that we have these stories to tell suggests that tumours of the central nervous system (CNS) are not as uncommon as many think. In fact, tumours of the CNS represent approximately $10 \%$ of all neoplasms and $2 \%$ of all cancer-related deaths per year. 'The most common primary brain tumours are the gliomas and the meningiomas, whereas cancer of the breast and lung are the cancers most likely to have metastases in the brain. Although it is of both academic and clinical value to be aware of the different psychiatric/neurological symptoms associated with different tumours and tumour locations, it is as important to remember that these are generalisations and that in the real world a patient with any tumour type in any location in the brain may potentially present with any psychiatric symptom. Furthermore, although there are some classic syndromes described with brain tumours, the presenting symptoms may be indistinguishable from those of a 'true' psychiatric illness.

The literature on the neuropsychiatry of brain tumours is relatively limited and the quality of the studies is often questionable, particularly from the point of view of the psychiatrist, who is primarily interested in knowing when to suspect a brain tumour in a patient presenting with psychiatric complaints. There is considerably more information available on the psychiatric symptoms that occur in series on patients known to have brain tumours. Therefore, although we have many retrospective studies of psychiatric symptoms in patients with brain tumours, there is a serious shortage of prospective studies.

In the classic study by Keschner et al., ${ }^{2} 530$ patients with diagnosed brain tumours were evaluated for psychiatric symptoms. This study found that up to $70 \%$ of patients with brain tumours had neuropsychiatric symptoms, with other studies giving even higher figures. The study by Keschner and co-workers $^{2}$ reported that $18 \%$ of brain tumour patients presented initially with psychiatric rather than neurological symptoms. This figure is supported by a recent paper by Gupta and Kumar, ${ }^{3}$ who found that $21 \%$ of their series presented with psychiatric symptoms only. Whereas neuropsychiatric symptoms are very common in patients with brain tumours, the prevalence of brain tumours in psychiatric patients is relatively low at between 1/200 and 1/1000, albeit considerably higher than in the general population. ${ }^{3}$ Interestingly, at least two postmortem series ${ }^{4,5}$ showed that about half of these tumours remain undiagnosed until the death and autopsy of the psychiatric patient.

\section{Factors affecting the clinical presentation of brain tumours}

Although any psychiatric symptom or constellation of symptoms is possible, there are certain 'rules of thumb' that may affect the clinical presentation of the patient with a brain tumour.

\section{Rate of growth}

The more rapidly a tumour expands, the more likely it is to cause psychiatric symptoms. Conversely, however, slowgrowing tumours (like meningiomas) may allow for more adaptive changes in the brain and therefore the tumour is more likely to be 'neurologically silent' and may present only with psychiatric symptoms without general or localising neurological symptoms and signs. In terms of psychiatric symptoms, fast-growing tumours are more likely to cause psychosis and agitation, while slow-growing tumours tend to cause personality changes, apathy, depression and subtle cognitive changes. ${ }^{6}$

\section{Location}

Supratentorial tumours are considerably more likely to cause psychiatric symptoms than infratentorial tumours. ${ }^{2,3}$ In fact, in one series ${ }^{7}$ patients with infratentorial tumours were completely devoid of psychiatric symptoms, in contrast to patients with tumours above the tentorium, $44 \%$ of whom had 
psychiatric symptoms. Also, tumours in the frontal and temporal lobes are more likely to cause psychiatric symptoms than those in parietal or occipital lobes. Left-sided, frontal tumours also seem to be associated with higher rates of depression, while those in the frontal lobe of the right hemisphere may be associated with features that may be considered 'manic'. ${ }^{8}$

\section{Pressure effects}

Tumours that cause raised intracranial pressure cause more psychiatric symptoms. Some have speculated that the level of symptoms may be related to oedema caused by the tumour in the surrounding brain tissue which may result in disruption of intracerebral pathways, rather than to the actual size of the tumour.?

\section{Premorbid factors}

The patient with a higher $I Q$ will be less impaired than the patient with lower intelligence. Equally, the well-adapted, psychologically healthy individual is likely to cope better with the diagnosis of a brain tumour than patients with premorbid psychological problems; to a large extent, level of integration will determine the psychological adaptive responses of the individual to the diagnosis.

\section{Tumour type}

Although there are some indications that the type of tumour may indeed have an effect on clinical presentation, this may only be a function of the location and rate of expansion. Meningiomas are more often found in the frontal areas of the brain, but are slower growing than the gliomas. Therefore patients with meningiomas may be more likely to present with psychiatric symptoms only. Tumours that cause multiple lesions are also more likely to cause psychiatric symptoms than single tumours. ${ }^{3,7,9}$

\section{Clinical clues that a brain tumour may be present}

Certain clinical symptoms and signs suggest the possibility of a space-occupying lesion in the brain of the psychiatric patient and should prompt further investigation. These are firstly, and obviously, the presence of any focal neurological signs. This once again underscores the need for thorough physical, and in particular neurological, examination of psychiatric patients, particularly those presenting for the first time or presenting with new symptoms. Similarly, new- onset seizures of any type should alert the physician to the possibility of structural abnormality in the brain of the patient. Headaches may also be the first presenting symptom of a brain tumour. The physician should be alerted by headaches that are of new onset, are positional or nocturnal, or occur on awakening. Nausea and vomiting, particularly when associated with headaches, may also be indicative of the pressure effects of a space-occupying lesion. The most 'psychiatric' indicators of possible brain tumour are sudden personality changes or the first onset of psychosis after the age of 45 years, and presentation with these symptoms is probably an indication for structural brain imaging. ${ }^{3}$

\section{The role of neuropsychology}

Neuropsychology forms an integral part of the ongoing evaluation of the patient with a brain tumour as well as the treatment and rehabilitative processes. Although neuropsychology is not really seen as a diagnostic tool any longer as it has been superseded by advanced structural and functional brain-imaging techniques, it is of great importance in determining the extent of the cognitive impairment caused by the tumour and the medical and surgical interventions that may follow diagnosis. A thorough neuropsychological evaluation will therefore provide a baseline against which future changes can be measured.

\section{Localising features of brain tumours}

As mentioned earlier, any tumour in any location in the brain may potentially cause any psychiatric symptom. Although it is often disputed, it seems that there are, however, certain classic symptoms and signs associated with tumour location in specific areas of the brain. ${ }^{10}$ In general, tumours are more likely to cause psychiatric symptoms when located supratentorially and in the frontal or medial lobes. Also, tumours of the left hemisphere seem to be more likely to cause psychotic symptoms, whereas tumours of the right hemisphere cause more affective symptoms.

\section{The frontal lobes}

Psychiatric symptoms are very common with tumours in this area. Although symptoms and signs often overlap, it is clinically useful to consider the frontal lobes in terms of three different areas that cause different symptom complexes. ${ }^{6}$ 


\section{Orbito-frontal area}

Patients with lesions in this area often present with what have been termed 'pseudopsychopathic' features, which may consist of any of the following: disinhibition, impulsiveness, inappropriate jocular affect and euphoria and emotional lability. These patients are often highly distractible and show poor insight into their impairment. The poor social and financial judgement that is often part of this syndrome may have dire consequences if not recognised early. A factor that may confuse the unskilled clinician is that patients often do not have obvious cognitive decline on clinical evaluation. Even with intelligence testing on standard test batteries, they may score within the normal range. The neuropsychologist who evaluates this patient will therefore have to pay particular attention to executive functions and structure the battery of tests in such a way that orbito-frontal functions are evaluated fully.

\section{Frontal convexity}

A tumour in this area may cause the patient to seem apathetic and indifferent, with psychomotor retardation and a picture that may be confused with major depressive disorder. In sharp contrast with this, these patients may have sudden, aggressive outbursts with no or minimal provocation. They often have elements of perseveration, poor word list generation, motor programming deficits, poor abstraction and poor visuospatial abilities.

\section{Medial aspects of frontal lobes}

A clinical picture with profound reduction in spontaneous movements and speech and even complete akinesis and mutism may develop. Such patients may have episodes of

\section{SADOC}

South African Doctors' Club International

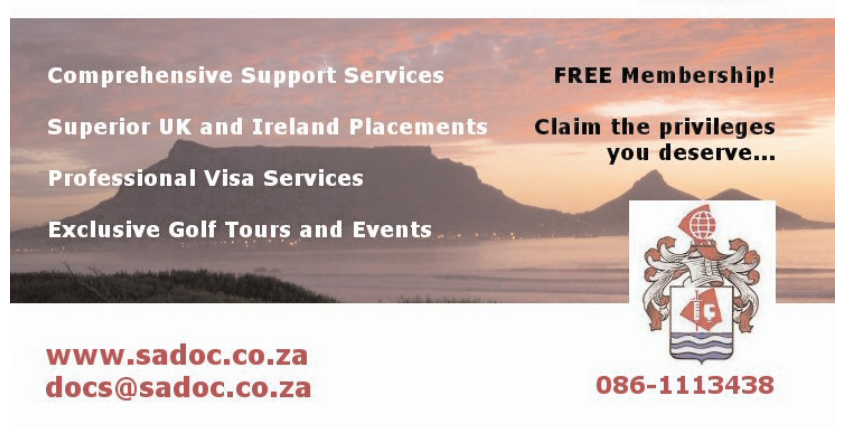

incontinence, and on neurological examination may be found to have weakness and/or loss of sensation in the lower extremities. Even when there is no clear weakness or hearing impairment, they may respond poorly, or not at all, to commands.

\section{The temporal lobes}

Seizures are common with lesions of this area. If the tumour is located in the dominant hemisphere the patient may have a fluent aphasia (Wernicke's aphasia) combined with what has been described as 'schizophrenia-like' symptoms. ${ }^{5}$ This refers to the presence of psychotic phenomena, most notably hallucinations. However there are usually atypical signs and symptoms to alert the clinician to the fact that the clinical presentation may not be that of schizophrenia. These include hallucinations other than the typical auditory hallucinations that we so often see in patients with schizophrenia and may include visual, tactile and olfactory hallucinations. Also, symptoms may come in 'spells' and may be associated with sudden mood swings or sudden, intrusive suicidal thoughts. Another factor distinguishing this condition from Diagnostic and Statistical Manual (DSM)-defined schizophrenia is the fact that the affect of these patients with temporal pathology is often well retained and warm, as opposed to the more or less blunted affect of so many patients with schizophrenia. On neuropsychological testing patients with temporal lobe tumours may have problems with verbal and non-verbal memory, may show reduced ability for new learning and may also have problems with visuo-spatial memory, particularly if the tumour is in the non-dominant hemisphere. "1

\section{The parietal lobes}

A tumour in this area may result in highly complex clinical phenomena, particularly in terms of cognitive disturbances, which are more likely to occur than psychiatric disturbances per se. ${ }^{6,10}$ Because the symptomatology may be so unusual, the clinician must be wary of not labelling these symptoms and signs as either conversion or a somatoform disorder. Patients with lesions in this area do not usually present with psychotic features. Mood symptoms that result from a tumour in this area are more likely to be depressive or apathetic, rather than manic in nature. If the tumour is in the dominant hemisphere the patient may also have dysphasia, ideomotor and/or ideational apraxia and some or all of the features of the so-called Gerstmann's syndrome, which consists of finger agnosia, dyscalculia, dysgraphia and right-left 
disorientation. With the tumour in the non-dominant parietal lobe, symptoms are more likely to include visuospatial deficits, dressing apraxia, astereognosis (inability to recognise familiar objects by touch), anosognosia (denial of illness), unilateral inattention, marked, inappropriate indifference and social isolation. ${ }^{12}$

\section{The occipital lobes}

This area of the brain is 'quieter' in terms of psychiatric features than either the frontal or temporal lobes. The most 'psychiatric' of symptoms with a tumour in this area are visual hallucinations. These are often simple hallucinations and not the complex hallucinations that one may find in certain psychiatric illnesses. Whereas psychiatric symptoms may be less prominent with tumours in this area, neuropsychological testing may show prominent impairment of cognitive and perceptual functions. This may also be associated with visual agnosia, homonymous hemianopia and prosopagnosia (inability to recognise familiar faces).

\section{Other fumours}

\section{Diencephalic tumours}

These will often involve the limbic system and are therefore likely to cause psychiatric symptoms. The neuropsychological deficits most commonly observed are problems with memory and new learning.

\section{Corpus callosum}

Tumours and other lesions in the corpus callosum (CC) are notorious for the severity of psychiatric symptoms that they cause. ${ }^{13}$ Tumours in this area often cause behavioural manifestations first. Tumours in the anterior part of the CC are more likely to cause psychiatric symptoms than those in the posterior part. Many different affective and/or psychotic symptoms as well as personality changes may be seen when the CC is involved. With involvement of the splenium severe impairment in memory and visual perception can be expected.

\section{Pituitary}

Tumours in this area often involve other diencephalic structures and may therefore cause psychiatric symptoms. Abnormalities of the physiological functions that are coordinated here can be expected, some of which may be misinterpreted as psychiatric symptoms. These include changes in sleep, temperature regulation and sexual functioning, among others.

\section{Conclusion}

Although brain tumours are relatively rare occurrences, they have a higher incidence in psychiatric patients than in the general population. In most cases, thorough mental state and neurological evaluation will alert the clinician to the possibility of a brain tumour; however this is not always the case. Clinicians do not have to do brain imaging in all patients, but should be particularly vigilant when symptoms occur for the first time after age 45, have a sudden onset or involve sudden changes in personality and behaviour.

\section{References}

Kurtzke JF. Neuroepidemiology. Ann Neurol 1984; 16: 265-277

2. Keschner M, Bender MB, Strauss I. Mental symptoms associated with brain tumour: a study of 530 verified cases. JAMA 1938; 110: 714-718.

3. Gupta RK, Kumar R. Benign brain tumours and psychiatric morbidity: a 5-years retrospective data analysis. Aust N ZJ Psychiatry 2004; 38: $316-319$.

4. Klotz M. Incidence of brain tumors in patients hospitalized for chronic mental disorders. Psychiatr Q 1957; 31: 669-680

5. Selecki BR. Intracranial space-occupying lesions among patients admitted to mental hospitals. Med J Aust 1965; 1: 383-390

6. Price TRP, Goetz KL, Lovell MR. Neuropsychiatric aspects of brain tumors. In: Yudovsky SC, Hales RE, eds. The American Psychiatric Press Textbook of Neuropsychiatry. Washington, DC: American Psychiatric Press, 1992: 473-497.

7. Lampl Y, Barak Y, Achiron A, Sarova Pinchas I. Intracranial meningiomas. Correlation of peritumoural oedema and psychiatric disturbances. Psychiatr Res 1995; 58: 177-180

8. Starkstein SE, Robinson RG, Price TR. Comparison of cortical and subcortical lesions in the production of post stroke mood disorders. Brain 1987; 110: 1045-1059.

9. Pringle AM, Taylor T, Whittle R. Anxiety and depression in patients with an intracranial neoplasm before and after tumour surgery. Br J Neurosurg 1999; 13: 46-51.

10. Lishman WA Cerebral tumours In: Lishman WA ed Organic Psychiatry The Psychological Consequences of Cerebral Disorder. Oxford: Blackwell Science, 1999: $218-236$

11. Butters N. Amnestic disorders. In: Heilman KM, Valenstein E, eds. Clinical Neuropsychology. New York: Oxford University Press, 1979: 439-474.

12. Critchley M. Psychiatric symptoms and parietal disease. Proceedings of the Royal Society of Medicine 1964; 57: 422-428.

13. von Rich hofen S TSGHMBU. Inte hemispheric transfer and its implications for neurology and psychiatry. Fortschr Neurol Psychiatr 2003; 71: 449-457. 